\title{
From Cultural Probes Tasks to Gamified Virtual Energy Missions
}

\author{
K. Samso \\ Dep. Matemàtiques i Informàtica \\ Gran via, 585, Bcn, 08007 Spain \\ kasamsomu7@ub.edu
}

D. Tellols

Dep. Matemàtiques i Informàtica

Gran via, 585, Bcn, 08007 Spain

dtelloas7@ub.edu

\author{
I. Rodríguez \\ Dep. Matemàtiques i Informàtica Dep. Matemàtiques i Informàtica \\ Gran via, 585, Bcn, 08007 Spain Gran via, 585, Bcn, 08007 Spain \\ inmarodriguez@ub.edu \\ annapuig@ub.edu \\ F. Escribano \\ Gamification Research Dep. \\ Gecon, St. Feliu de LI. Bcn \\ fescribano@gecon.es
}

This paper proposes a gamification design of a Cultural Probes (CP) in the context of the Smart Grid. CP is a well-known $\mathrm{HCl}$ technique used to inform and inspire applications design. Gamification aims to enhance the participation of users in CP tasks. Participants are children who develop tasks to provide designers with information about families' habits of energy consumption, and their knowledge and interest in energy related issues. We use a narrative that engages the player in the mission of saving people wasting energy crazily, under the influence of an evil wizard.

\section{Cultural Probes, Gamification, Energy awareness}

\section{INTRODUCTION}

In the context of the Smart Grid ${ }^{1}$, there is room to develop applications to inform prosumers (productors \& consumers) about their use of electricity, the state of the grid such as real time prices and peaks, give them useful recommendations to change habits, and even let them sell their own energy.

Nevertheless, the design of Smart Grid applications requires a sound knowledge of prosumers' needs, values and habits. Since user research techniques like interviews and direct observation can result intrusive, we advocate for the use of Cultural Probes (CP) (Gaver et al. (1999)) to gather data on energy information and energy management in families.

Cultural Probes technique was conceived consisting of a package with artifacts (camera, diary) and tasks, which was given to participants to record events, feelings and interactions. Afterwards, CP was also deployed as mobile applications. However, and whatever form it takes,

1 The Smart Grid is a technology-based electricity network that is used to supply electricity to prosumers using a two-way digital communication between the utility and them.
$\mathrm{CP}$ relies on participants being responsible and remembering to complete tasks at the assigned time and as instructed, which results on one of the drawbacks of cultural probes: the lack of participation of users, sometimes returning back the CP almost empty. It is in this context that we place the main hypothesis of our research, Gamification (i.e the use of game elements in nongame contexts) of cultural probes may encourage a more active and fruitful participation of users.

Therefore, the objective of our research is twofold. First, to demonstrate that the Gamification (i.e the use of game elements in non-game contexts) of CP may provide good results in terms of users' involvement and consequently in terms of number of tasks completed. And second, to use the CP to get valuable insight on families' energy-related habits in order to inform the design of an application that provides family members with detailed information about their use of electricity and so give them useful recommendations to change their bad habits. Collaterally, we think that the $\mathrm{CP}$ can raise awareness on energy and environmental issues.

Specifically, this paper focuses on the gamification design of our previously proposed cultural probe of electric energy designed for children (Tellols et al. (2016)). We propose to deploy the CP as a mobile 
application and use GMC (Gamification Model Canvas) (Jimenez and Escribano (2017)) design framework to guide the gamification process.

\section{RELATED WORK}

The gamification of the Smart Grid has already been considered (BarriosO'Neill and Hook (2016))(Marques and Nixon(2013)) and there are energy companies that have developed gamified applications to engage and motivate their customers in order to save energy (Oracle(2016)). However, in the context of energy consumption and lifestyle, gamification in a deep level (not only using PBL Itextendash Points, Badges and Leaderboards(textendash) is not easy since designers need to get into the emotional side of users and in their habits. That is why we propose $\mathrm{CP}$ to get valuable and meaningful insight on prosumers.

Since their proposal (Gaver et al.(1999)), CP has been used in many projects in order to obtain information about the user's behaviour. Our proposal differs from this classical approach as we give the CP to children in order to get data not only from them, but also from those they live with.

Research using CP with children has already been conducted following the classical approach (Wyeth and Diercke (2006) Gennari et al. (2016)), and digital CP involving the use of mobile phones, where the device was used for taking photos and recording audio clips (Iversen and Nielsen (2003)). We combine ideas of previous approaches and want to take full advantage of mobile apps and gamification to foster the participation of children in the energy $\mathrm{CP}$.

\section{CULTURAL PROBES FOR THE SMART GRID}

Participants are voluntary children between 10 and 12 years old who receive a 'digital package' (mobile app). A consent form must be signed by their parents, who will be informed about data anonymity and the use ${ }^{2}$ of data only for research purposes. In the following, we describe CP tasks and what we aim to obtain through each of them.

- Become a psychologist: analyze your family's usage habits by asking them which electrical appliances they use the most and their mood while using them. Through this task we intend to get emotional information to guide us on the design of how to influence the change of habits. Depending on the mood while using an appliance, tips

2 Thanks to the ethics commission of our institution for the approval and consent of the study. to save energy through that appliance can be more or less effective.

- Become a detective: observe energyrelated behaviour of each member of your family for a day and indicate (from a predefined list of behaviours) what they did. Through this task, we designers can see the correlation between the usage emotions they expressed on previous CP task and the correctness (i.e bad or good) of that usage. According to that, it can be easy to influence change on those bad usages where the mood is negative and necessary to explore other less or non electric alternatives on those where the mood is positive, as well as it could be interesting to prize and try to maintain good behaviours.

Collateral to data gathering for designers, this task may raise children awareness on energy saving and its environmental impact, as the children may learn what behaviours are more correct than others. Moreover, they can share what they learned with their relatives, influencing a change on them at the same time.

- Become an electrician: indicate the electrical appliances you have in each room in a house map. At the end of each day, mark those appliances that have been used and how much. Thanks to this task we can learn about the electrical appliances owned by families, their distribution and frequency of use. This helps to obtain data about family's habits as a whole, instead of individually, which complements the information extracted on the first and second CP task. Moreover, we make children aware of the usage of energy at home, so they can reflect on it and identify bad or good habits.

- Become a journalist: interview each member of your family. This task can be used for designers to gather data about families' needs of information about their use of electric energy, their preferences and predisposition to change habits. Also, it helps to obtain information about what knowledge members of the family have about energy consumption, the Smart Grid and green behaviour (through a short semistructured interview done by children).

Next section describes the gamification process of these $\mathrm{CP}$ tasks to encourage and engage $\mathrm{CP}$ participants.

\section{GAMIFICATION DESIGN OF THE CP}


We use the gamification design framework named GMC (Gamification Model Canvas), that has been applied by more than 16,000 gamificators from more than 180 different countries (Jimenez and Escribano (2017)). In the following, we specify our design referring to the nine elements proposed by the framework.

Revenues: Economic or social return of the solution. With the gamification of the CP we hope to achieve that children complete an elevated number of CP tasks by incrementing the participation and, therefore, the main goal is to obtain as much information as possible.

Simplicity: Problems and obstacles the users have to face. It should be critical to effectively engage children in the very beginning of the CP (break the ice). Moreover, kids could not have much free time due to both curricular and extracurricular activities, and have a low planning capacity (e.g reminders could help).

Players: Who and what the people are like. Participants of the CP are children from 8 to 12 years old. They can be considered newbies in the gamified system and, as proposed by GMC framework, their corresponding player types may be consumers, exploiters and killers, those mainly (but not only) driven by extrinsic motivations (Dixon (2011)). Moreover, kids will have to be willing and excited to perform this kind of dynamic activity.

Behaviours: Actions necessary to develop in our players. Participants have to develop certain actions in order to get meaningful insight for our purpose, which means to complete all subtasks of the main CP tasks (see previous section).

Aesthetics: Desirable emotional responses evoked in the player. We want players to be responsible of finishing $\mathrm{CP}$ tasks, and to be challenged and motivated to explore the system and discover new missions. Furthermore, their engagement could improve their family bonding (fellowship) achieving also children's discovery and awareness on it.

In order to grab their attention for our purposes, we propose to use a narrative, i.e. a story to motivate them to complete the gamified CP tasks, which is one of the main game mechanics agreed in the literature (Deterding et al. (2011) Dubbelman (2016)).

Performing all the tasks in the defined missions, children get information about people's behaviour

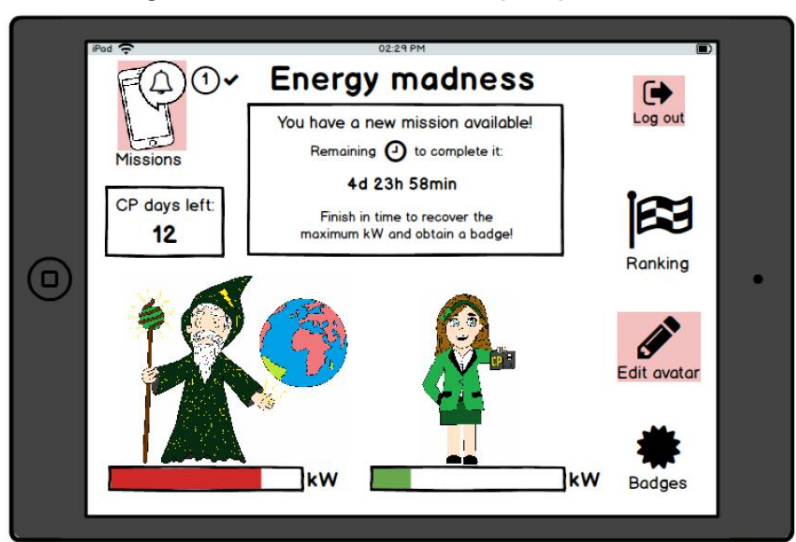

and, at the same time, they can help them to be more caring to the environment. Every time a mission is completed, few people is saved from the spell and kWh are taken away from the wizard (See Figure 1).

\section{Figure 1: Wireframe of the app dashboard.}

Complete wireframe can be found in ${ }^{3}$

Dynamics: Run-time behaviour of the mechanics acting on the player over time.

- Progression to give feedback to players through different levels and enhance the feeling of advance in the narrative towards the goal. The existence of a final goal (defeat the wizard) and the visualization of the progress (how far is the end) engage the user to finish the tasks to achieve that end. It would not happen in a scenario without a final objective, so we can say that this dynamic is very important in order to structure the process in the users' mind and motivate them.

- Status visualization in relation to the rest of players with the help of a ranking. In line with the above classification on player type, a dynamic such as status visualization, compared to others, will especially motivate the killer players.

- Feeling of identity through the owning of a customized avatar. Being able to customize the avatar generates a user's identity projection to it, creating or increasing an emotional link to the avatar. This helps to keep the user connected to the gamified system.

- Winning rewards. We all like to win the prize (extrinsic motivation) or acquire that social identity linked to the prize. Again, being able to win rewards is an element that increases the motivation of the players and keeps their attention focused on the goal.

- Getting reminders. Reminding the players to be constant and responsible with their appointment we compensate their lack of planning previously commented.

Components: Elements of the game to create mechanics or to give feedback.

- Missions (equivalent to the CP tasks) allow players to achieve the main goal of the game.

3

https://dl.dropboxusercontent.com/content_link /SrFWfMjxpvP5iL8hay0F3dfjuEOTUMtfMt76K3z mV7Xhr7cvJOlsFDNNlpglxQAW/file 
- The progress system is based on points $(\mathrm{kWh})$. There is a progress bar to give direct feedback to the players about their current status on the $\mathrm{CP}$, and a leader board with participants ranking. Missions' tasks completed in-time give a maximum reward to the player, meanwhile tasks performed out of time give a smaller reward.

- Badges acknowledge the tasks completed on time and quantity.

- Daily rewards or surprise gifts are random, to motivate children to interact with the application daily.

- An avatar represents the participant in the app with a customized image. They can access to virtual goods consisting of different elements to customize the avatar (including received gifts).

- The CP itself has a limited duration, so a global countdown is a visible element in the app.

- Moreover, and to facilitate participants' planning, certain $C P$ tasks have a local countdown, so they need to be accomplished in time to be get a badge and obtain a maximum number of $\mathrm{kWh}$.

- At the end of the CP, children with the first positions in the ranking receive a real physical prize, which would be an additional motivation to complete all the tasks and be more competitive (that they can check their position in the ranking at real time).

- Notifications remind the participants to complete the tasks.

Mechanics: Rules of the game with components for creating game dynamics. The CP game consists of four levels/missions. We consider the $\mathrm{CP}$ is over all the missions are completed, and the player who recovers more $\mathrm{kWh}$ from the wizard is the winner.

The game starts with the child taking the role of a special agent, after the registration (where players need to indicate 4 members of their family, preferably people who live with them) and avatar creation have been done successfully. As an agent, the child has to accept a set of 4 missions implicating to disguise as different characters.

In one of the missions players play the role of a psychologist and make four associations (personappliance-mood) of family member by asking an electrical appliance they frequently use and their mood while using it. They obtain points (recover $\mathrm{kWh}$ ) for each association made, and if they complete the mission in-time they receive a psychologist's diploma (badge) (see Figure 2 and

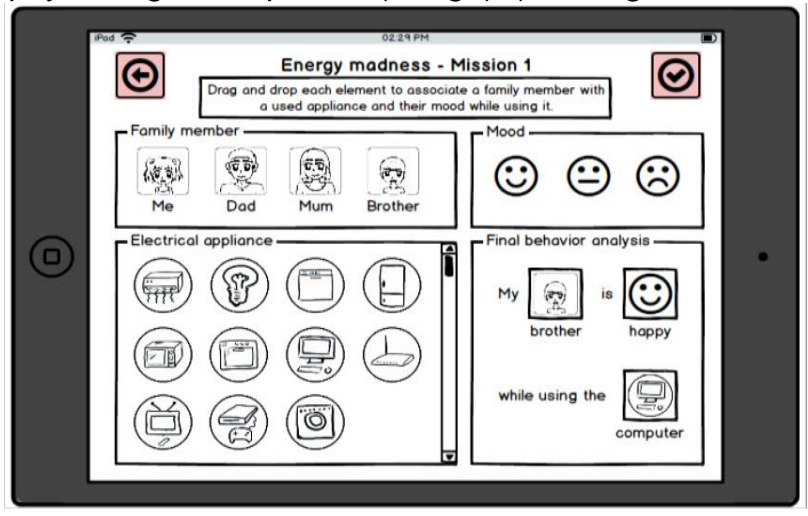

3).

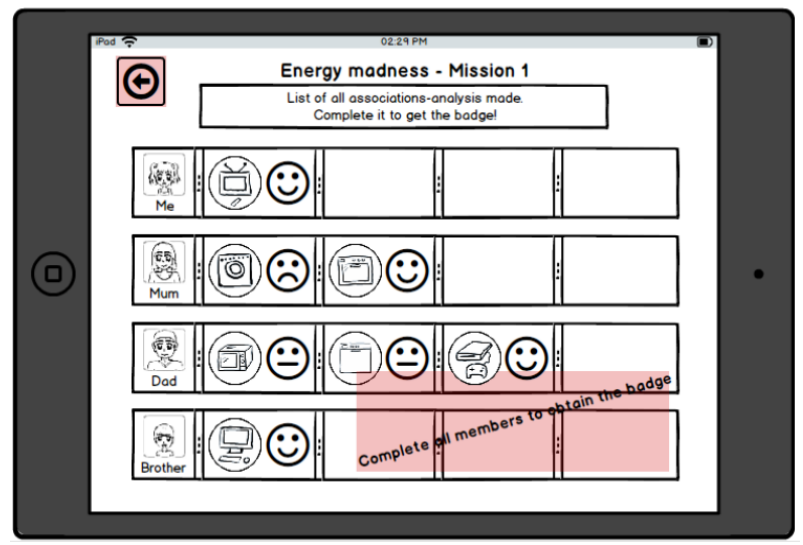

Figure 2: Psychologist Mission. Person-moodappliance association.

Figure 3: List of associations: Person-moodappliance

A detective is the role played in another mission. Players need to "observe" each member of their family and indicate what they did from a list of green and non green activities (saving or wasting energy actions, respectively). The more observed members, the more kWh recovered, and if players observe them all, they gain a detective's badge consisting in a pair of binoculars.

Another players' mission is to be an electrician (Figure 4 and Figure 5). They are asked to indicate the rooms and electrical appliances of their home in a house map. At the end of a day, participants have to select the used appliances that day and, after that, how much they were used. Daily notifications recall the mission to players, who recover $\mathrm{kWh}$ from the wizard for each completed day. Those players who complete the 2 requested days (a weekday and a weekend day) in-time earn some electrician's tools. 


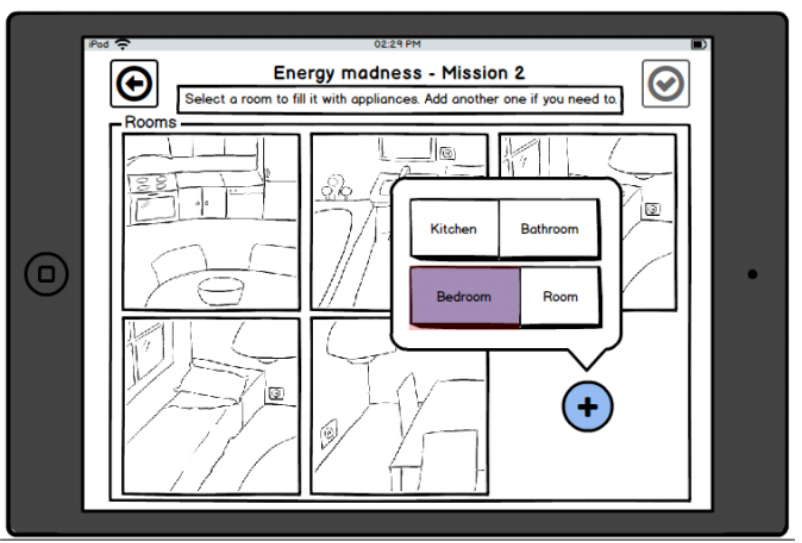

Figure 4: Electrician mission: rooms to fill with

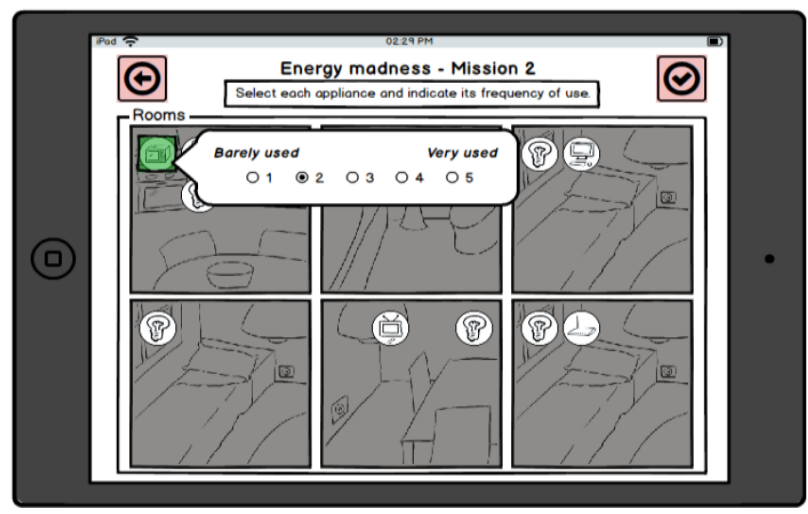

appliances

Figure 5: Electrician mission: frequency of use of appliances

Lastly, another mission the player acts as a journalist. It consists in interviewing each member

of the family, with a series of questions and answer choices, in order to know their preferences of information about energy saving and the Smart Grid. The more interviewed members, the more $\mathrm{kWh}$ the wizard loses. If all members are interviewed in-time, players achieve a press accreditation (badge). Once the duration of the CP is over (therefore, the time given to complete all the missions runs out), the game ends.

Platforms: Platforms on which to implement game mechanics. The app is designed to work on mobile devices (phone or tablet) in Android or iOS. This way, we can reach more public and the tracking and gathering of data can be done more efficiently.

\section{CONCLUSIONS}

We propose the design of a digital and gamified Cultural Probe (CP) in the context of the Smart Grid. The ultimate goals of the research are: i) demonstrate that gamified CP shows better results than non-gamified ones, and ii) inform the design of an application to provide information about energy use and give recommendations on energy savings and good habits.
Children, involved in a narrative with the goal of defeating a villain, help to gather information about families' electric consume and, at the same time, raise own and family members awareness on energy and environmental issues.

As on-going work, we are implementing the proposed gamified design and plan to evaluate it with groups of children interacting with two different versions (gamified/non-gamified) of the application. The main goal is to compare results between gamified CP and non-gamified CP. To do so, we'll take measures of task completion in both groups, both at the higher level of the CP (the 4 tasks/missions we asked to be completed) and at the finer level of each task/mission. In the same line of thought, we want to know the percentage of tasks started but not finished and also tasks left in blank in both experimental conditions. Additionally, we want to gather children opinions related to the entire experience, such as how much they enjoyed $\mathrm{CP}$ activities and what's their perception of own and family's level of awareness in energy-related issues before and after performing the CP.

Lessons learned with this research can also be applied to CP in other family-related matters such as water consumption and waste management.

\section{ACKNOWLEDGEMENTS}

Thanks to research projects TIN2015-66863-C2-1$\mathrm{R}$ (MINECO/FEDER), research funding 2014SGR623, Rubi Brilla, schools Montesori and 25 setembre in Rubi, and Escola del Mar in Barcelona for their availability for user evaluation in the very near-term.

\section{REFERENCES}

Barrios-O'Neill, D. and A. Hook (2016). Future energy networks and the role of interactive gaming

as simulation. Futures $81,119-129$. Modelling and Simulation in Futures Studies.

Deterding, S., D. Dixon, R. Khaled, and L. Nacke (2011). From game design elements to gamefulness: Defining "gamification". In Proceedings of the 15th International Academic MindTrek Conference: Envisioning Future Media Environments,

MindTrek '11, New York, NY, USA, pp. 9-15. ACM.

Dixon, D. (2011). Player types and gamification. In Proceedings of the CHI 2011 Workshop Gamification: Using Game Design Elements in Non-Game context, pp. 41-43. ACM.

Dubbelman, T. (2016). Narrative game mechanics. In Interactive Storytelling. International 
Conference on Interactive Digital Storytelling 2016.

Lecture Notes in Computer Science. Springer.

Gaver, B., T. Dunne, and E. Pacenti (1999, January).

Design: Cultural probes. Interactions 6(1), 2129.

Gennari, R., A. Melonio, and S. Torello (2016).

Gamified probes for cooperative learning: a case

study. Multimedia Tools and Applications, 1-25.

Iversen, O. S. and C. Nielsen (2003).

Using

digital cultural probes in design with children.

In Proceedings of the 2003 Conference on

Interaction Design and Children, IDC '03, New

York, NY, USA, pp. 154-154. ACM.

Jimenez,

$\mathrm{S}$.

and

$\mathrm{F}$.

Escribano

(2017).

Gamification model canvas.

Visited on

January,

2017 from

http://gecon.es/wp-

content/uploads/2016/0/gamification model canvas v02.pdf.

Marques, B. and K. Nixon (2013, Sept).

The

gamified grid: Possibilities for utilising gamebased

motivational psychology to empower the smart social grid. In 2013 Africon, pp. 1-5.

Oracle (2016). opower. Visited on December 23, 2016 from https://opower.com.

Tellols, D., K. Samso, I. Rodriguez, and A. Puig (2016). Cultural probes for the gamification of energy awareness. ACM WomENcourage 2016.

Wyeth, P. and C. Diercke (2006).

Designing

cultural probes for children.

In Proceedings

of the 18th Australia Conference on ComputerHuman Interaction: Design: Activities, Artefacts and Environments, OZCHI '06, New York, NY,

USA, pp. 385-388. ACM. 